\title{
Refining the criteria for immediate total-body CT after severe trauma
}

\author{
Kaij Treskes ${ }^{1}$ (D) - Teun P. Saltzherr ${ }^{2} \cdot$ Michael J. R. Edwards $^{3}$ - Benn J. A. Beuker ${ }^{4}$. Esther M. M. Van Lieshout ${ }^{5}$. \\ Joachim Hohmann ${ }^{6}$ • Jan S. K. Luitse ${ }^{1}$. Ludo F. M. Beenen ${ }^{7}$ - Markus W. Hollmann ${ }^{8}$ - Marcel G. W. Dijkgraaf ${ }^{9}$. \\ J. Carel Goslings ${ }^{1,10}$ - on behalf of the REACT-2 study group
}

Received: 10 June 2019 / Revised: 8 September 2019 / Accepted: 8 October 2019/Published online: 23 January 2020

(C) The Author(s) 2020

\begin{abstract} diagnostic approach, proper selection of the severely injured patients is mandatory. The revised set retains $8.78 \mathrm{mSv}$ (95\% CI 6.01-11.56) for 36\% of the non-severely injured patients. value for severe injury and reduces radiation exposure for less severely injured patients.

Key Points

- Selection criteria for iTBCT can be reduced to 10 clinically useful criteria.

- This reduces radiation exposure in $36 \%$ of less severely injured patients.

- Overall discriminative capacity for selection of severely injured patients remained equal.
\end{abstract}

Objectives Initial trauma care could potentially be improved when conventional imaging and selective CT scanning is omitted and replaced by immediate total-body CT (iTBCT) scanning. Because of the potentially increased radiation exposure by this

Methods In the REACT-2 trial, severe trauma patients were randomized to iTBCT or conventional imaging and selective CT based on predefined criteria regarding compromised vital parameters, clinical suspicion of severe injuries, or high-risk trauma mechanisms in five trauma centers. By logistic regression analysis with backward selection on the 15 study inclusion criteria, a revised set of criteria was derived and subsequently tested for prediction of severe injury and shifts in radiation exposure.

Results In total, 1083 patients were enrolled with median ISS of 20 (IQR 9-29) and median GCS of 13 (IQR 3-15). Backward logistic regression resulted in a revised set consisting of nine original and one adjusted criteria. Positive predictive value improved from $76 \%$ (95\% CI $74-79 \%$ ) to $82 \%$ (95\% CI $80-85 \%$ ). Sensitivity decreased by $9 \%$ (95\% CI $7-11 \%$ ). The area under the receiver operating characteristics curve remained equal and was 0.80 (95\% CI $0.77-0.83$ ), original set 0.80 (95\% CI 0.77-0.83).

Conclusions Selection criteria for iTBCT can be reduced from 15 to 10 clinically criteria. This improves the positive predictive

Electronic supplementary material The online version of this article (https://doi.org/10.1007/s00330-019-06503-2) contains supplementary material, which is available to authorized users.

Kaij Treskes

k.treskes@amsterdamumc.nl

1 Trauma Unit, Department of Surgery, Amsterdam University Medical Centers, location AMC, Meibergdreef 9, 1105 AZ Amsterdam, the Netherlands

2 Department of Surgery, Haaglanden Medical Center, Lijnbaan 32, 2512 VA Den Haag, the Netherlands

3 Trauma Unit, Department of Surgery, Radboud University Medical Center, Geert Grooteplein-Zuid 10, 6525 GA Nijmegen, the Netherlands

4 Trauma Unit, Department of Surgery, University Medical Center Groningen, Hanzeplein 1, 9700 RB Groningen, the Netherlands

5 Trauma Research Unit, Department of Surgery, Erasmus MC, University Medical Center Rotterdam, 's-Gravendijkwal 230, 3015 CE Rotterdam, the Netherlands
6 Department of Radiology and Nuclear Medicine, University of Basel Hospital, Petersgraben, 4031 Basel, Switzerland

7 Department of Radiology, Amsterdam University Medical Centers, location AMC, Meibergdreef 9, 1105 AZ Amsterdam, the Netherlands

8 Department of Anaesthesiology, Amsterdam University Medical Centers, location AMC, Meibergdreef 9, 1105 AZ Amsterdam, the Netherlands

9 Clinical Research Unit/Department of Clinical Epidemiology, Biostatistics and Bioinformatics, Amsterdam University Medical Centers, location AMC, University of Amsterdam, Meibergdreef 9, 1105 AZ Amsterdam, the Netherlands

10 Department of Surgery, Onze Lieve Vrouwe Gasthuis, Jan Tooropstraat 164, 1061 AE Amsterdam, the Netherlands 
Keywords Multiple trauma $\cdot$ Wounds and injuries $\cdot$ Diagnostic imaging $\cdot$ Multidetector computed tomography $\cdot$ Whole-body imaging

$\begin{array}{ll}\text { Abbreviations } \\ \text { AIS } & \text { Abbreviated injury score } \\ \text { CI } & \text { Confidence interval } \\ \text { CT } & \text { Computed tomography } \\ \text { GCS } & \text { Glasgow coma scale } \\ \text { IQR } & \text { Interquartile range } \\ \text { ISS } & \text { Injury severity score } \\ \text { iTBCT } & \text { Immediate total-body CT } \\ \text { PPV } & \text { Positive predictive value } \\ \text { ROC } & \text { Receiver operating characteristics } \\ \text { SBP } & \text { Systolic blood pressure } \\ \text { STWU } & \text { Standard workup }\end{array}$

\section{Introduction}

Improvements in speed and accuracy of computed tomography (CT) made immediate total-body CT (iTBCT) feasible as a diagnostic tool in the primary care for severe trauma patients. Initial trauma care for severe trauma patients can be improved when the step-up approach of conventional imaging and selective CT is omitted and an iTBCT is performed instead. iTBCT scanning is safe, shortens the time to end of imaging, and does not increase direct medical costs [1]. However, it has not been demonstrated to improve survival [1]. Because of the potentially increased radiation exposure by this diagnostic approach, proper selection of severely injured patients is mandatory [2-4]. Criteria for total-body CT in trauma vary across trauma centers and consensus is lacking [5, 6]. Early identification of severely injured patients will reduce exposure to radiation by iTBCT in less severely injured patients.

The decision to perform an iTBCT is based on information obtained during the pre-hospital phase and during the inhospital primary survey. Justification for performing an iTBCT is only possible in hindsight, when radiologic imaging, interventions, and the clinical course have confirmed all diagnoses. The REACT-2 was a randomized controlled trial setup to determine the effect of iTBCT on mortality compared to conventional imaging and selective CT. Inclusion criteria of this multicenter randomized trial aimed to select severely injured patients benefitting most from iTBCT before imaging [7].

The aim of the present analysis was to assess the discriminatory power of REACT-2 criteria for severely injured patients that could benefit from iTBCT during the primary assessment of trauma care. Furthermore, a revised set of criteria was derived and tested for discriminatory characteristics on detection of severe injury and shifts in radiation exposure compared to the original set of REACT-2 inclusion criteria.

\section{Materials and methods}

\section{Study design and patient selection}

This study is a secondary analysis of the REACT-2 trail in which non-pregnant adult severe trauma patients were included in five trauma centers in the Netherlands and Switzerland between April 2011 and January 2014. Inclusion was based on predefined compromised vital parameters, clinical suspicion of specific severe injuries, and high-risk trauma mechanisms. Patients were considered eligible when meeting one or more of the 15 inclusion criteria and none of the exclusion criteria as shown in Table 6 in the Appendix.

Patients were randomized to iTBCT or the standard workup (STWU) that consists of conventional imaging with selective CT of specific body regions (i.e., head, neck, chest and/or abdomen, and pelvis). Decision of eligibility by the trauma leader as well as documentation of the concerning criteria by a trauma team member was performed before the start of radiologic imaging. After obtaining vital parameters, a physical examination, and potentially life-saving interventions (e.g., securing airway, chest tube placement, or hemorrhage control measures), the trauma team proceeded to $\mathrm{CT}$ scanning in the same or an adjacent trauma resuscitation room. CT scanning could be interrupted any moment when the patient should deteriorate and could be reached within seconds by trauma team members. iTBCT was performed without preceding conventional imaging and consisted of an unenhanced $\mathrm{CT}$ of the head and neck with arms alongside the trunk. The second part consisted of a contrast enhanced CT of chest, abdomen, and pelvis. The preferred technique of the second part was splitbolus intravenous contrast imaging with the arms raised alongside the head [8]. Brain reconstruction was in axial planes with 5-mm head kernel and 1-mm bone kernel, cervical spine in 1-mm bone kernel in axial, sagittal, and coronal planes. Torso was reconstructed at 3-mm axial and coronal slices in soft and bone kernel. CT scanners at the participating sites were all 64-slice multidetector row CT scanners. Indication for selective $\mathrm{CT}$ of specific body regions was set by local protocols.

The design of the REACT-2 study has been previously described (ClinicalTrials.gov: NCT01523626) and published [7]. The REACT-2 study was approved by the medical ethics committees at all participating centers (AMC MEC 10/145).

\section{Outcome}

iTBCT was considered justified if a patient was classified as severely injured by in-hospital findings and clinical course. 
Definition of severe injury in the current study was met by presence of at least one of the following conditions:

- $\quad$ Injury Severity Score (ISS) $\geq 16$

- Requiring emergency surgery or emergency radiologic intervention

- Direct admission to the intensive care unit

- In-hospital death

\section{Statistical analysis}

Continuous data with a normal distribution are presented as means with standard deviation and non-normally distributed data are presented as medians with interquartile ranges. Independent sample $t$ tests and Mann-Whitney $U$ tests were used to compare the parametric and non-parametric continuous data, respectively. The chi-square test was used to compare the categorical variables. A $p$ value of less than 0.05 was considered statistically significant.

To identify criteria that could select severely injured patients, we entered all REACT-2 inclusion criteria in backward stepwise multivariate logistic regression analysis on severe injury using $p<0.05$ as criterion. These criteria are clinically useful and available early in the primary trauma assessment. Selection by univariate logistic regression analysis on single REACT-2 inclusion criteria before the multivariate analysis was omitted since the criteria were defined in advance. Thereby, there were more events or non-events (i.e., status as severely injured patient or status as non-severely injured patient) present in the study population than 10 -fold the 15 REACT-2 inclusion criteria, which allowed multivariate analysis of all criteria. When clinically appropriate, the threshold values for vital parameters and trauma mechanism characteristics of specific criteria were retrospectively adjusted and included again in the regression analysis. Threshold value for pulse was increased by steps of 10 per minute, for systolic blood pressure (SBP) lowered by steps of $10 \mathrm{mmHg}$, and for fall from height by steps of $1 \mathrm{~m}$. Positive predictive value (PPV), relative sensitivity, and receiver operating characteristics (ROC) were used to compare the accuracy of the sets of criteria.

Numbers needed to iTBCT scan to perform one unnecessary iTBCT scan for a non-severely injured patient were compared between the sets of criteria, calculated by $(1 /(1-\mathrm{PPV}))$. Reduction of iTBCT scans for non-severely injured patients was calculated by subtraction of false positive rates ( $1-\mathrm{PPV})$. Shifts in radiation exposure were calculated by subtraction of the sum of all effective doses from all radiological examinations done in the trauma room. The radiation dose was estimated based on the dose catalog of Mettler and colleagues [9]. Differences of the mean for radiation doses were presented with $95 \%$ CI. All statistical analyses were performed with SPSS version 24 (SPSS Inc.).

\section{Results}

In the REACT-2 trial, 1083 patients were enrolled of which $541(50.0 \%)$ underwent iTBCT as primary diagnostic modality. Within the entire group, 785 patients $(72.5 \%)$ eventually underwent TBCT during the primary assessment as they underwent an iTBCT or CT scans from the head, neck, chest, abdomen, and pelvis secondary to x-rays and ultrasound. Median age was 43 (IQR 27-59) and 76\% of the patients were male. Median ISS was 20 (IQR 9-29) and median in-hospital Glasgow Coma Scale (GCS) was 13 (IQR 3-15). Baseline demographic and clinical characteristics are presented in Table 1.

There were 827 severely injured patients as defined by the combined outcome and therefore the original set of criteria has a PPV for severe injury of $76 \%$ (95\% CI 74-79\%). Table 2 presents the prevalence within the enrolled population and the PPV for each separate criterion. Backward logistic regression analysis of the 15 original criteria resulted in selection of seven criteria shown in Table 3. After adjustment of threshold values for vital parameters and trauma mechanism characteristics, the backward selection resulted in nine original and one adjusted criteria. Therefore, five of the original criteria (respiratory rate $\geq 30 / \mathrm{min}$ or $\leq 10 / \mathrm{min}$, pulse $\geq 120 / \mathrm{min}$, ejection form a vehicle, death of occupant in same vehicle, and severely injured patient in same vehicle) were not of additional value and can be omitted.

Table 4 shows that PPV of the newly formed set of criteria statistically significantly increased to $82 \%$ (95\% CI 80-85\%) compared to $76 \%$ (95\% CI 74-79\%) of the original set. Sensitivity of the revised set within the originally formed population was statistically significantly reduced by $9 \%(95 \%$ CI 7-11\%). Clinical characteristics including trauma scores comparing severely injured patients not selected by the revised set of criteria to selected severely injured patients are displayed in Table 8 in the Appendix. The area under the ROC curve remained equal and was $0.80(95 \% \mathrm{CI} 0.77-0.83)$ in the revised set compared to 0.80 (95\% CI $0.77-0.83$ ) for the original set as shown in Figure 1 in the Appendix. Numbers of iTBCT scans needed to perform one unnecessary scan for a non-severely injured patient was statistically significantly improved from 1 in 4.2 (95\% CI 3.8-4.7) to 1 in 5.6 (95\% CI 4.9-6.5). The number of unnecessary iTBCT scans was statistically significantly decreased with $6 \%(95 \%$ CI $2-10 \%)$.

Shifts in radiation exposure for the different sets of criteria are displayed separately for severely injured and non-severely injured patients in Table 5. With the use of the original criteria, iTBCT adds $1.19 \mathrm{mSv}$ (95\% CI - 0.13-2.51) for severely injured patients and $8.15 \mathrm{mSv}$ (95\% CI 5.91-10.39) for non- 
Table 1 Baseline demographic and clinical characteristics, $n_{\max }=1083$

\begin{tabular}{|c|c|c|}
\hline Characteristic & $n^{*}$ & \\
\hline Age (years) & 1083 & $43(27-59)$ \\
\hline Male sex, $n(\%)$ & 1083 & $824(76.1)$ \\
\hline Blunt trauma, $n(\%)$ & 1083 & $1064(98.2)$ \\
\hline Trauma mechanism blunt trauma, $n(\%)$ & 1064 & \\
\hline Fall from height & & $348(32.7)$ \\
\hline MVC - patient as occupant & & $391(36.7)$ \\
\hline MVC - patient as cyclist & & $125(11.7)$ \\
\hline MVC - patient as pedestrian & & $74(7.0)$ \\
\hline Other & & $126(11.8)$ \\
\hline \multicolumn{3}{|l|}{ Pre-hospital vital parameters } \\
\hline Respiratory rate (per minute) & 640 & $16(14-20)$ \\
\hline Pulse (bpm) & 948 & $89(25)^{\dagger}$ \\
\hline Systolic blood pressure (mmHg) & 910 & $133(31)^{\dagger}$ \\
\hline GCS (points) & 1061 & $14(6-15)$ \\
\hline Triage Revised Trauma Score & 618 & $7.04(5.03-7.84)$ \\
\hline \multicolumn{3}{|l|}{ In-hospital vital parameters } \\
\hline Respiratory rate (per minute) & 669 & $16(14-20)$ \\
\hline Pulse (bpm) & 1059 & $88(22)^{\dagger}$ \\
\hline Systolic blood pressure $(\mathrm{mmHg})$ & 1060 & $131(27)^{\dagger}$ \\
\hline Hypotensive at admission, $n(\%)$ & - & $82(7.7)$ \\
\hline GCS (points) & 1083 & $13(3-15)$ \\
\hline Revised Trauma Score & 651 & $7.11(4.09-7.84)$ \\
\hline Total-body CT, $n(\%)$ & 1083 & $785(72.5)$ \\
\hline Immediate total-body CT, $n(\%)$ & 1083 & $553(51.1)$ \\
\hline Abbreviated Injury Scale $\geq 3, n(\%)$ & 1083 & \\
\hline Head & & $465(42.9)$ \\
\hline Chest & & $435(40.2)$ \\
\hline Abdomen & & $116(10.7)$ \\
\hline Extremities & & $304(28.1)$ \\
\hline Injury Severity Score (points) & 1083 & $20(9-29)$ \\
\hline Multitrauma patients, $n(\%)^{\ddagger}$ & 1083 & $693(64.0)$ \\
\hline TBI patients, $n(\%)^{*}$ & 1083 & $329(30.4)$ \\
\hline TRISS, survival probability & 618 & $0.94(0.68-0.98)$ \\
\hline
\end{tabular}

Results of the population described in this table were published earlier [1]. All data are number (\%) or median (interquartile range) unless otherwise specified

*This column displays the number of patients that was analyzed for each specific variable

${ }^{\dagger}$ Mean (SD)

* Multitrauma patients are defined as ISS $\geq 16$. TBI patients are defined as GCS $<9$ at presentation and AIS head $\geq 3$

MVC motor vehicle collision, $C T$ computed tomography, TBI traumatic brain injury, TRISS Trauma and Injury Severity Score

severely injured patients compared to the STWU. Within patients not selected for iTBCT by the revised criteria, the STWU retains $1.32 \mathrm{mSv}(95 \% \mathrm{CI}-2.71-5.35)$ for $9 \%$ of the severely injured patients and retains $8.78 \mathrm{mSv}(95 \% \mathrm{CI}$
$6.01-11.56$ ) for $36 \%$ of the non-severely injured patients compared to iTBCT. Shifts in radiation exposure are displayed separately for age groups $<45$ years and $>45$ years in Tables 8 and 9 in the Appendix.

\section{Discussion}

By retrospective analysis of a prospectively formed cohort of severe trauma patients, we derived a revised set of 10 criteria for iTBCT, shown in Table 6. The new set of criteria has an increased PPV for detecting severe injury. Hence, these criteria could reduce the number of patients screened by iTBCT who are less severely injured and who will not have an advantage of all their body regions scanned. The relative reduction of sensitivity compared to the original set could be restrained to $9 \%$. This reduction of sensitivity leads to a relative increase of severely injured patients for whom screening by iTBCT will be retained and will have conventional imaging and selective CT scanning. Since there is no reduction of mortality after iTBCT for the trial population selected by the original criteria, the aim for a revised set of iTBCT criteria with higher PPV and lower sensitivity can be justified. Without loss of overall discriminative capacity for severe injuries, we changed the set of criteria for iTBCT with emphasis on the reduction of radiation exposure for the less severely injured patient.

Quantification of the shifts in radiation exposure was performed separately for the less severely injured patients. For $36 \%$ of the less severely injured patient, a significant reduction in radiation exposure could be demonstrated by use of the revised set of criteria. This effect was also present for patients of age $<45$ years. The precise amounts of reduction in radiation exposure have to be interpreted in perspective of ongoing developments of low-dose CT scanning.

Compromised vital parameters, clinical suspicion of severe injuries, and high-risk mechanisms are widely used as criteria for TBCT in severe trauma $[5,6]$. The first report by Wurmb et al on such a set of criteria for iTBCT described a PPV of $69 \%$ and sensitivity of $97 \%$ for ISS $\geq 16$ in sedated and ventilated severe trauma patients. The difference in outcome measure and the selection of sedated and ventilated patients makes the results difficult to compare to our study [10]. Hsiao et al reported $32 \%$ PPV and 50\% sensitivity of criteria for TBCT by clinical judgment for the presence of multi-region injury defined by an Abbreviated Injury Score (AIS) of $\geq 2$ in two or more body regions. After retrospective identification of predictors for multi-region injury, a prediction model was made that did not show improvement for the area under the ROC curve compared to indication by clinical judgment [11].

Hemodynamically compromised patients could benefit from trauma screening by iTBCT. Wada et al [12] reported reduced mortality for patients receiving $\mathrm{TBCT}$ before 
Table 2 Predictive value of REACT-2 immediate total-body CT criteria for severe injuries, $n=1083$

$n \quad \mathrm{PPV}, \%(95 \% \mathrm{CI}) \quad \mathrm{NPV}, \% *(95 \% \mathrm{CI}) \quad \mathrm{Sens}, \% *(95 \% \mathrm{CI}) \quad \mathrm{Spec}, \% *(95 \% \mathrm{CI})$

Parameters at hospital arrival

\begin{tabular}{|c|c|c|c|c|c|}
\hline Respiratory rate $\geq 30 / \mathrm{min}$ or $\leq 10 / \mathrm{min}$ & 16 & $81(62-100)$ & $24(21-26)$ & $2(1-2)$ & $99(98-100)$ \\
\hline Pulse $\geq 120 / \mathrm{min}$ & 69 & $80(70-89)$ & $24(21-27)$ & $7(5-8)$ & $95(92-97)$ \\
\hline Pulse $\geq 130 / \mathrm{min}^{\dagger}$ & 49 & $88(79-97)$ & $24(22-27)$ & $5(4-7)$ & $98(96-100)$ \\
\hline Pulse $\geq 140 / \mathrm{min}^{\dagger}$ & 26 & $88(63-76)$ & $24(21-27)$ & $3(2-4)$ & $99(98-100)$ \\
\hline Systolic blood pressure $\leq 100 \mathrm{mmHg}$ & 116 & $96(92-99)$ & $26(23-29)$ & $13(11-16)$ & $98(96-100)$ \\
\hline Systolic blood pressure $<90 \mathrm{mmHg}^{\dagger}$ & 82 & $100(100-100)$ & $26(23-28)$ & $10(8-12)$ & $100(100-100)$ \\
\hline Systolic blood pressure $<80 \mathrm{mmHg}^{\dagger}$ & 32 & $100(100-100)$ & $24(22-27)$ & $4(3-5)$ & $100(100-100)$ \\
\hline Estimated exterior blood loss $\geq 500 \mathrm{ml}$ & 43 & $91(82-99)$ & $24(22-27)$ & $5(3-6)$ & $98(97-100)$ \\
\hline GCS $\leq 13$ or abnormal pupillary reaction & 485 & $93(91-95)$ & $37(33-41)$ & $55(51-58)$ & $87(83-91)$ \\
\hline $\mathrm{GCS} \leq 8^{\dagger}$ & 437 & $99(98-100)$ & $39(35-43)$ & $52(49-56)$ & $98(97-100)$ \\
\hline $\mathrm{GCS}=3^{\dagger}$ & 394 & $99(99-100)$ & $37(33-41)$ & $47(44-51)$ & $99(98-100)$ \\
\hline \multicolumn{6}{|l|}{ Clinical suspicions } \\
\hline Fractures from at least two long bones & 90 & $89(82-95)$ & $25(22-28)$ & $10(8-12)$ & $96(94-99)$ \\
\hline Flail chest, open chest, or multiple rib fractures & 114 & $83(76-90)$ & $25(22-27)$ & $12(9-14)$ & $93(89-96)$ \\
\hline Severe abdominal injury & 65 & $82(72-91)$ & $24(21-27)$ & $6(5-8)$ & $95(93-98)$ \\
\hline Pelvic fracture & 98 & $78(69-86)$ & $24(21-27)$ & $9(7-11)$ & $91(88-95)$ \\
\hline Unstable vertebral fractures/spinal cord compression & 69 & $68(57-79)$ & $23(21-26)$ & $6(4-7)$ & $91(88-95)$ \\
\hline \multicolumn{6}{|l|}{ Injury mechanisms } \\
\hline Fall from height $(>3 \mathrm{~m} />10 \mathrm{ft})$ & 319 & $62(57-67)$ & $18(15-20)$ & $24(21-27)$ & $53(47-59)$ \\
\hline Fall from height $(>4 \mathrm{~m} />13 \mathrm{ft})^{\dagger}$ & 166 & $70(64-77)$ & $23(20-25)$ & $14(12-17)$ & $81(76-86)$ \\
\hline Fall from height $(>5 \mathrm{~m} />16 \mathrm{ft})^{\dagger}$ & 126 & $71(64-79)$ & $23(20-26)$ & $11(9-13)$ & $86(82-90)$ \\
\hline Fall from height $(>6 \mathrm{~m} />20 \mathrm{ft})^{\dagger}$ & 82 & $78(69-87)$ & $24(21-26)$ & $8(6-10)$ & $93(90-96)$ \\
\hline Fall from height $(>7 \mathrm{~m} />23 \mathrm{ft})^{\dagger}$ & 60 & $87(78-95)$ & $24(22-27)$ & $6(5-8)$ & $97(95-99)$ \\
\hline Fall from height $(>8 \mathrm{~m} />26 \mathrm{ft})^{\dagger}$ & 40 & $88(77-98)$ & $24(22-27)$ & $4(3-6)$ & $98(96-100)$ \\
\hline Ejection from a vehicle & 30 & $60(42-78)$ & $23(21-26)$ & $2(1-3)$ & $95(93-98)$ \\
\hline Death of occupant in same vehicle & 17 & $65(42-87)$ & $24(21-26)$ & $1(1-2)$ & $98(96-100)$ \\
\hline Severely injured patient in same vehicle & 18 & $78(59-97)$ & $24(21-26)$ & $2(1-3)$ & $98(97-100)$ \\
\hline Wedged or trapped chest/abdomen & 60 & $83(74-93)$ & $24(21-27)$ & $6(4-8)$ & $96(94-99)$ \\
\hline
\end{tabular}

*Within the group of patients selected by the original criteria

${ }^{\dagger}$ Retrospectively adjusted criteria

GCS Glasgow Coma Scale, $P P V$ positive predictive value, NPV negative predictive value, Sens sensitivity, Spec specificity, $C I$ confidence interval

emergency bleeding control measurements in a retrospective study in two trauma centers. Reduction in mortality in trauma patients requiring emergency bleeding control interventions by iTBCT could not be confirmed in the REACT-2 population. However, a potentially clinically relevant absolute risk reduction of $11.2 \%$ (95\% CI - 0.3 to $22.7 \%$ ) in comparison with the STWU was observed [13]. Huber-Wagner et al [14] reported reduced mortality in severe trauma patients in moderate (SBP 90-110 $\mathrm{mmHg}$ ) or severe (SBP < $90 \mathrm{mmHg}$ ) shock when receiving TBCT during the resuscitation in a retrospective multicenter study. In the present study, compromised blood pressure (SBP $<100 \mathrm{mmHg}$ ) is an independent predictor for severe injury and is therefore a valid indication for iTBCT. It is recommendable to only perform CT scanning on hemodynamically compromised patients in the trauma resuscitation room or the adjacent room and the trauma team has direct access to the patient and has options for potential life-saving interventions any moment.

Patients with a compromised GCS could benefit from trauma screening by TBCT. Kimura et al [15] reported reduced mortality in patients with moderate to severe consciousness disturbance (GCS 3-12) in a retrospective multicenter study. Furthermore, decreased levels of consciousness could be considered an indication on itself since several clinical indicators for imaging are unreliable owing to the lack of subjective input from the patient when screening for injuries. Routine CT imaging for patients with unreliable physical examination is reported to reveal unsuspected findings in up to $38 \%$, leading to treatment changes in 19-26\% [16, 17]. Our study found GCS $\leq 13$ or abnormal pupillary reaction an independent 
Table 3 Predictive value of REACT-2 immediate total-body CT criteria for severe injuries, $n=1083$

\begin{tabular}{|c|c|c|c|c|c|c|c|}
\hline & \multirow{2}{*}{\multicolumn{3}{|c|}{ Univariate analysis }} & \multicolumn{4}{|c|}{ Backward selection of criteria } \\
\hline & & & & \multicolumn{2}{|l|}{ Original criteria } & \multicolumn{2}{|l|}{ Adjusted criteria } \\
\hline & $n$ & OR $(95 \% \mathrm{CI})$ & $p$ & OR $(95 \% \mathrm{CI})$ & $p$ & OR $(95 \% \mathrm{CI})$ & $p$ \\
\hline \multicolumn{8}{|l|}{ Parameters at hospital arrival } \\
\hline Respiratory rate $\geq 30 / \mathrm{min}$ or $\leq 10 / \mathrm{min}$ & 16 & $1.35(0.38-4.76)$ & 0.644 & - & - & - & - \\
\hline Pulse $\geq 120 / \min$ & 69 & $1.23(0.67-2.25)$ & 0.499 & - & - & - & - \\
\hline Pulse $\geq 130 / \mathrm{min}^{*}$ & 49 & $2.29(0.96-5.43)$ & 0.061 & & & - & - \\
\hline Pulse $\geq 140 / \mathrm{min}^{*}$ & 26 & $2.41(0.72-8.10)$ & 0.154 & & & - & - \\
\hline Pulse (continuous) $)^{\dagger}$ & & $1.02(1.01-1.02)$ & $<0.001$ & & & - & - \\
\hline Systolic blood pressure $\leq 100 \mathrm{mmHg}$ & 116 & $7.78(3.14-19.29)$ & $<0.001$ & $5.71(2.23-14.62)$ & $<0.001$ & $5.72(2.22-14.75)$ & $<0.001$ \\
\hline Systolic blood pressure $<90 \mathrm{mmHg} *$ & 82 & $\infty(0-\infty)$ & 0.996 & & & & \\
\hline Systolic blood pressure $<80 \mathrm{mmHg} *$ & 32 & $\infty(0-\infty)$ & 0.998 & & & & \\
\hline Systolic blood pressure (continuous) ${ }^{\dagger}$ & & $0.99(0.98-0.99)$ & $<0.001$ & & & & \\
\hline Estimated exterior blood loss $\geq 500 \mathrm{ml}$ & 43 & $3.12(1.10-8.81)$ & 0.032 & $3.29(1.09-9.93)$ & 0.035 & $3.70(1.20-11.37)$ & 0.023 \\
\hline GCS $\leq 13$ or abnormal pupillary reaction & 485 & $7.83(5.32-11.52)$ & $<0.001$ & $10.02(6.69-15.00)$ & $<0.001$ & $12.65(8.23-19.45)$ & $<0.001$ \\
\hline $\mathrm{GCS} \leq 8^{*}$ & 437 & $69.24(25.54-187.66)$ & $<0.001$ & & & & \\
\hline $\mathrm{GCS}=3 *$ & 394 & $114.45(28.28-463.28)$ & $<0.001$ & & & & \\
\hline GCS (continuous) $)^{\dagger}$ & & $0.69(0.64-0.74)$ & $<0.001$ & & & & \\
\hline \multicolumn{8}{|l|}{ Clinical suspicions } \\
\hline Fractures from at least two long bones & 90 & $2.64(1.34-5.16)$ & 0.005 & $4.08(2.02-8.25)$ & $<0.001$ & $4.94(2.41-10.15)$ & $<0.001$ \\
\hline $\begin{array}{l}\text { Flail chest, open chest, or multiple rib } \\
\text { fractures }\end{array}$ & 114 & $1.62(0.97-2.71)$ & 0.066 & $2.81(1.62-4.86)$ & $<0.001$ & $3.27(1.85-5.76)$ & $<0.001$ \\
\hline Severe abdominal injury & 65 & $1.39(0.73-2.65)$ & 0.313 & - & - & $2.18(1.07-4.42)$ & 0.031 \\
\hline Pelvic fracture & 98 & $1.08(0.66-1.77)$ & 0.771 & $1.76(1.03-3.01)$ & 0.039 & $1.82(1.05-3.14)$ & 0.033 \\
\hline $\begin{array}{l}\text { Unstable vertebral fractures/spinal cord } \\
\text { compression } \\
\text { Injury mechanisms }\end{array}$ & 69 & $0.64(0.38-1.09)$ & 0.098 & - & - & $1.87(1.06-3.31)$ & 0.032 \\
\hline Fall from height $(>3 \mathrm{~m} />10 \mathrm{ft})$ & 319 & $0.35(0.26-0.47)$ & $<0.001$ & - & - & - & - \\
\hline Fall from height $(>4 \mathrm{~m} />13 \mathrm{ft})^{*}$ & 166 & $0.70(0.48-1.01)$ & 0.054 & & & $1.64(1.07-2.52)$ & 0.022 \\
\hline Fall from height $(>5 \mathrm{~m} />16 \mathrm{ft})^{*}$ & 126 & $0.75(0.49-1.13)$ & 0.167 & & & & \\
\hline Fall from height $(>6 \mathrm{~m} />20 \mathrm{ft})^{*}$ & 82 & $1.11(0.65-1.91)$ & 0.709 & & & & \\
\hline Fall from height $(>7 \mathrm{~m} />23 \mathrm{ft}) *$ & 60 & $2.08(0.98-4.44)$ & 0.058 & & & & \\
\hline Fall from height $(>8 \mathrm{~m} />26 \mathrm{ft})^{*}$ & 40 & $2.22(0.86-5.72)$ & 0.099 & & & & \\
\hline Fall from height (continuous) ${ }^{\dagger}$ & & $1.17(1.06-1.29)$ & 0.002 & & & & \\
\hline Ejection from a vehicle & 30 & $0.45(0.22-0.95)$ & 0.037 & - & - & - & - \\
\hline Death of occupant in same vehicle & 17 & $0.56(0.21-1.53)$ & 0.261 & - & - & - & - \\
\hline Severely injured patient in same vehicle & 18 & $1.09(0.35-3.33)$ & 0.887 & - & - & - & - \\
\hline Wedged or trapped chest/abdomen & 60 & $2.71(1.04-4.54)$ & 0.038 & $2.11(1.00-4.42)$ & 0.049 & $2.57(1.20-5.51)$ & 0.015 \\
\hline
\end{tabular}

* Retrospectively adjusted criteria

${ }^{\dagger}$ Continuous data of regarding criterion used were possible

GCS Glasgow Coma Scale, OR odds ratio, CI confidence interval

predictor for severe injury and further supports a compromised GCS to be a valid indication for iTBCT after severe trauma.

Besides vital parameters that indicate a hemodynamically or neurologically compromised status, also clinical suspicions of specific injuries and high-risk trauma mechanisms independently predict patients to be severely injured in our study. Although these criteria are prone to interpretation differences, we would recommend adopting these criteria in iTBCT indication schemes. During mass casualty accidents, overruling the iTBCT indication scheme has to be considered [18, 19]. Furthermore, there should be awareness for the increase of incidental findings by TBCT compared to the STWU during implementation or refining of iTBCT indication schemes [20, 21]. 
Table 4 Characteristics of different sets of criteria for immediate total-body CT

\begin{tabular}{llllll}
\hline & PPV (95\% CI) & $\begin{array}{l}\text { Relative sensitivity* } \\
(95 \% \text { CI })\end{array}$ & ROC AUC (95\% CI) & $\begin{array}{l}\text { Numbers needed } \\
\text { to overscan }^{\dagger}(95 \% \text { CI })\end{array}$ & $\begin{array}{l}\text { Decrease of unnecessary } \\
\text { iTBCT scans }\end{array}$ \\
\hline Original criteria $(n=15)$ & $76 \%(74-79)$ & Reference & $0.80(0.77-0.83)$ & $4.2(3.8-4.7)$ & Reference \\
Selected original criteria $(n=7)$ & $87 \%(85-90)$ & $80 \%(77-83)$ & $0.78(0.75-0.81)$ & $7.9(6.7-9.8)$ & $11 \%(8-14)$ \\
Selected adjusted criteria $(n=10)$ & $82 \%(80-85)$ & $91 \%(89-93)$ & $0.80(0.77-0.83)$ & $5.6(4.9-6.5)$ & $6 \%(2-10)$ \\
\hline
\end{tabular}

* Relative sensitivity within the population preselected by the original criteria

${ }^{\dagger}$ Number of iTBCT scans to perform one unnecessary iTBCT for a non-severely injured patient

${ }^{\ddagger}$ Percentage decrease of iTBCT scans for non-severely injured patients

$R O C$ receiver operating characteristic, $A U C$ area under the curve, $P P V$ positive predictive value, $C I$ confidence interval

\section{Limitations and strengths}

The main limitation of this study is the lack of information of patients who were not selected by the original REACT-2 criteria for eligibility of screening by iTBCT. This study could therefore only report the relative reduction of the sensitivity by the revised set compared to the original set of criteria. If proportions of severely injured patients in the group not selected by the original criteria were available, the absolute sensitivity, specificity, and negative predictive value could have been calculated. The proposed revised set of iTBCT criteria should be prospectively validated in another cohort of patients.

The definition of multitrauma and multi-region injured patients is subject of debate. Several cut-off values for ISS or AIS are used with eventual involvement of vital parameters proposed [22]. As a part of the combined outcome measure of this study, we chose ISS $\geq 16$ to justify iTBCT in hindsight for patients with multiple relevant injuries (AIS $\geq 3$ in two or more body regions or AIS $\geq 3$ in one body region and AIS $\geq 2$ in two or more body regions) and patients with a severe injury of at least one body region (AIS $\geq 4$ ). Hsiao et al $[11]$ chose AIS $\geq 2$ in two regions as the anatomical outcome measure to justify TBCT. In our opinion, TBCT for patients with eventually AIS of 2 in two body regions is not justified. On the contrary, the screening of a patient with a severe injury in only one body region could be justified since there is a higher probability of concomitant injury, which should be quickly excluded with high accuracy.

An alternative approach for refining the criteria for iTBCT criteria is to determine its discriminative power for selection of patients who would otherwise receive equal or even higher radiation exposure by selective $\mathrm{CT}$ scanning compared to the radiation exposure of iTBCT. This particularly reflects the judgment of the trauma team leader for the necessity of CT scans of specific body regions which does not necessarily correlates with selection of severely injured patients [23]. Therefore, the radiation exposure by the diagnostic approach with selective CT scans was not eligible as outcome measure for revision of the iTBCT criteria.

Strength of this multicenter study is the assessment of prospectively observed criteria for iTBCT in a large trial population. Previous studies assessed retrospectively observed TBCT criteria or were performed in a single-center setting. The combined clinical outcome parameter is suitable to define severely injured patients and patients that need fast and detailed diagnostics when an immediate intervention or ICU treatment is indicated. The addition of immediate surgery to

Table 5 Shifts in radiation exposure in different sets of criteria for immediate total-body CT and standard workup with selective CT, $n=1083$

\begin{tabular}{|c|c|c|c|c|c|c|}
\hline & \multicolumn{2}{|l|}{ Original criteria (15) } & \multicolumn{2}{|l|}{ Selected criteria (7) } & \multicolumn{2}{|c|}{ Selected and adjusted criteria (10) } \\
\hline & $\begin{array}{l}\text { Additional radiation } \\
\text { exposure compared to } \\
\text { STWU, mSv (95\% CI) }\end{array}$ & $\begin{array}{l}\% \text { of } \\
\text { population }\end{array}$ & $\begin{array}{l}\text { Additional radiation } \\
\text { exposure compared to } \\
\text { STWU, mSv (95\% CI) }\end{array}$ & $\begin{array}{l}\% \text { of } \\
\text { population }\end{array}$ & $\begin{array}{l}\text { Additional radiation } \\
\text { exposure compared to } \\
\text { STWU, mSv (95\% CI) }\end{array}$ & $\begin{array}{l}\% \text { of } \\
\text { population }\end{array}$ \\
\hline \multicolumn{7}{|l|}{ Selected for iTBCT } \\
\hline Severely injured & $1.19(-0.13$ to 2.51$)$ & 76.4 & $2.05(0.56$ to 3.53$)$ & 60.9 & $1.17(-0.23$ to 2.57$)$ & 69.7 \\
\hline \multirow[t]{2}{*}{ Non-severely injured } & 8.15 (5.91 to 10.39$)$ & 23.6 & $7.24(3.07$ to 11.41$)$ & 8.8 & 7.91 (4.77 to 11.05$)$ & 15.1 \\
\hline & $\begin{array}{l}\text { Additional radiation } \\
\text { exposure compared to } \\
\text { iTBCT, mSv }(95 \% \mathrm{CI})\end{array}$ & $\begin{array}{l}\% \text { of } \\
\text { population }\end{array}$ & $\begin{array}{l}\text { Additional radiation } \\
\text { exposure compared to } \\
\text { iTBCT, } \mathrm{mSv}(95 \% \mathrm{CI})\end{array}$ & $\begin{array}{l}\% \text { of } \\
\text { population }\end{array}$ & $\begin{array}{l}\text { Additional radiation } \\
\text { exposure compared to } \\
\text { iTBCT, } \mathrm{mSv}(95 \% \mathrm{CI})\end{array}$ & $\begin{array}{l}\% \text { of } \\
\text { population }\end{array}$ \\
\hline \multicolumn{7}{|l|}{ Selected for STWU } \\
\hline Severely injured & - & - & $2.11(-0.74$ to 4.95$)$ & 15.4 & $-1.32(-5.35$ to 2.71$)$ & 6.7 \\
\hline Non-severely injured & - & - & $-8.78(-11.44$ to -6.13$)$ & 14.9 & $-8.78(-11.56$ to -6.01$)$ & 8.5 \\
\hline
\end{tabular}

iTBCT immediate total-body CT, STWU standard workup with selective CT 
Table 6 Revised criteria for immediate total-body CT in trauma patients

Trauma patients with one of the following parameters at hospital arrival:

- Systolic blood pressure $<100 \mathrm{mmHg}$

- Estimated exterior blood loss $\geq 500 \mathrm{ml}$

- Glasgow Coma Score $\leq 13$ or abnormal pupillary reaction

AND/OR

Patients with a clinical suspicion of one of the following diagnoses:

- Fractures from at least two long bones

- Flail chest, open chest, or multiple rib fractures

- Severe abdominal injury

- Pelvic fracture

- Unstable vertebral fractures/spinal cord compression

AND/OR

Patients with one of the following injury mechanisms:

- Fall from a height (>4 m/>13 ft)

- Wedged or trapped chest/abdomen

Contra indications*

Trauma patients with one of the following characteristics:

- Known age $<18$ years

- Known pregnancy

- Referred from another hospital

- Clearly low-energy trauma with blunt injury mechanism

- Any patient with a stab wound in one body region

- Any patient who is judged to be too unstable to undergo a CT scan and requires (cardiopulmonary) resuscitation or immediate operation because death is imminent

*Contra indications for immediate total-body CT were not revised. These criteria are mentioned in this table to give a complete overview

the combined outcome measure is supported by reports of potential time and survival benefit for patients receiving emergency surgery $[12,24]$. The revised set of criteria will reduce the exposure to radiation for less severely injured patients without loss of discriminative capacity for severe injury. Thereby, the revision led to a simplification, which implies easier application during primary trauma care.

\section{Conclusion}

This study presents a revised set of 10 clinically criteria for iTBCT with a high predictive value for severe injury and therefore reduces radiation for the less severely injured patients for iTBCT. The criteria selected as predictors in this study should be prospectively validated in another cohort of patients for whom screening by iTBCT is considered after severe trauma.

Funding information The REACT-2 has received funding by ZonMw, the Netherlands Organisation for Health Research and Development (grant number: 171102023).

\section{Compliance with ethical standards}

Guarantor The scientific guarantor of this publication is Prof. J.C. Goslings.

Conflict of interest The authors of this manuscript declare no relationships with any companies whose products or services may be related to the subject matter of the article.

Statistics and biometry Prof. M.G.W. Dijkgraaf has significant statistical expertise.

Informed consent Written informed consent was obtained from all subjects (patients) in this study.

Ethical approval Institutional Review Board approval was obtained.

Study subjects or cohorts overlap All study subjects have been reported earlier in an article reporting the main outcome measures of the REACT-2 trial (Sierink JC, Treskes K, Edwards MJ et al Immediate total-body CT scanning versus conventional imaging and selective CT scanning in patients with severe trauma (REACT-2): a randomized controlled trial. Lancet. 2016;388(10045):673-83).

\section{Methodology}

- Prospective

- Diagnostic or prognostic study

- Multicenter study

Collaborators J.C. Sierink, S. van Dieren, V.M. de Jong, D. Den Hartog, T. Hagenaars, G.S.R. Muradin, R. Bingisser, C. Zähringer, N. Bless, R. van Vugt, T.N. Tromp, M. Brink, K. ten Duis, J.S. Harbers, K.W. Wendt.

Open Access This article is distributed under the terms of the Creative Commons Attribution 4.0 International License (http:// creativecommons.org/licenses/by/4.0/), which permits unrestricted use, distribution, and reproduction in any medium, provided you give appropriate credit to the original author(s) and the source, provide a link to the Creative Commons license, and indicate if changes were made.

\section{References}

1. Sierink JC, Treskes K, Edwards MJ et al (2016) Immediate totalbody $\mathrm{CT}$ scanning versus conventional imaging and selective $\mathrm{CT}$ scanning in patients with severe trauma (REACT-2): a randomised controlled trial. Lancet 388:673-683

2. Stengel D, Frank M, Matthes G et al (2009) Primary pan-computed tomography for blunt multiple trauma: can the whole be better than its parts? Injury 40(Suppl 4):S36-S46

3. Asha S, Curtis KA, Grant N et al (2012) Comparison of radiation exposure of trauma patients from diagnostic radiology procedures before and after the introduction of a panscan protocol. Emerg Med Australas 24:43-51

4. Sierink JC, Saltzherr TP, Wirtz MR, Streekstra GJ, Beenen LF, Goslings JC (2013) Radiation exposure before and after the introductionof a dedicated total-body CT protocolin multitrauma patients. Emerg Radiol 20:507-512

5. Hinzpeter R, Boehm T, Boll D et al (2017) Imaging algorithms and $\mathrm{CT}$ protocols in trauma patients: survey of Swiss emergency 
centers. Eur Radiol 27:1922-1928. https://doi.org/10.1007/s0033000016-04574-00331

6. Treskes K, Saltzherr TP, Luitse JS, Beenen LF, Goslings JC (2017) Indications for total-body computed tomography in blunt trauma patients: a systematic review. Eur J Trauma Emerg Surg 43:35-42

7. Sierink JC, Saltzherr TP, Beenen LF et al (2012) A multicenter, randomized controlled trial of immediate total-body $\mathrm{CT}$ scanning in trauma patients (REACT-2). BMC Emerg Med 12:4

8. Beenen LF, Sierink JC, Kolkman S et al (2015) Split bolus technique in polytrauma: a prospective study on scan protocols for trauma analysis. Acta Radiol 56:873-880. https://doi.org/10.1177/ 0284185114539319

9. Mettler FA Jr, Huda W, Yoshizumi TT, Mahesh M (2008) Effective doses in radiology and diagnostic nuclear medicine: a catalog. Radiology 248:254-263. https://doi.org/10.1148/radiol. 2481071451

10. Wurmb TE, Fruhwald P, Hopfner W, Roewer N, Brederlau J (2007) Whole-body multislice computed tomography as the primary and sole diagnostic tool in patients with blunt trauma: searching for its appropriate indication. Am J Emerg Med 25:1057-1062

11. Hsiao KH, Dinh MM, McNamara KP et al (2013) Whole-body computed tomography in the initial assessment of trauma patients: is there optimal criteria for patient selection? Emerg Med Australas 25:182-191

12. Wada D, Nakamori Y, Yamakawa K et al (2013) Impact on survival of whole-body computed tomography before emergency bleeding control in patients with severe blunt trauma. Crit Care 17:R178

13. Treskes K, Saltzherr TP, Edwards MJR et al (2019) Emergency bleeding control interventions after immediate total-body CT scans in trauma patients. World J Surg 43:490-496. https://doi.org/10. 1007/s00268-00018-04818-00260

14. Huber-Wagner S, Biberthaler P, Haberle S et al (2013) Whole-body $\mathrm{CT}$ in haemodynamically unstable severely injured patients-a retrospective, multicentre study. PLoS One 8:e68880

15. Kimura A, Tanaka N (2013) Whole-body computed tomography is associated with decreased mortality in blunt trauma patients with moderate-to-severe consciousness disturbance: a multicenter, retrospective study. J Trauma Acute Care Surg 75:202-206
16. Self ML, Blake AM, Whitley M, Nadalo L, Dunn E (2003) The benefit of routine thoracic, abdominal, and pelvic computed tomography to evaluate trauma patients with closed head injuries. Am J Surg 186:609-613

17. Salim A, Sangthong B, Martin M, Brown C, Plurad D, Demetriades D (2006) Whole body imaging in blunt multisystem trauma patients without obvious signs of injury: results of a prospective study. Arch Surg 141:468-473

18. Berger FH, Korner M, Bernstein MP et al (2016) Emergency imaging after a mass casualty incident: role of the radiology department during training for and activation of a disaster management plan. Br J Radiol 89:20150984. https://doi.org/10.1259/bjr. 20150984

19. Young VS, Eggesbo HB, Gaarder C, Naess PA, Enden T (2017) Radiology response in the emergency department during a mass casualty incident: a retrospective study of the two terrorist attacks on 22 July 2011 in Norway. Eur Radiol 27:2828-2834. https://doi. org/10.1007/s00330-00016-04677-00338

20. Mortani Barbosa EJ Jr, Osuntokun O (2019) Incidental findings in thoracic CTs performed in trauma patients: an underestimated problem. Eur Radiol 06313-06316

21. Treskes K, Bos SA, Beenen LFM et al (2017) High rates of clinically relevant incidental findings by total-body CT scanning in trauma patients; results of the REACT-2 trial. Eur Radiol 27:24512462. https://doi.org/10.1007/s00330-00016-04598-00336

22. Butcher N, Balogh ZJ (2009) The definition of polytrauma: the need for international consensus. Injury 40(Suppl 4):S12-S22

23. Shannon L, Peachey T, Skipper N et al (2015) Comparison of clinically suspected injuries with injuries detected at whole-body CT in suspected multi-trauma victims. Clin Radiol 70:1205-1211. https://doi.org/10.1016/j.crad.2015.1206.1084

24. Wurmb TE, Quaisser C, Balling H et al (2011) Whole-body multislice computed tomography (MSCT) improves trauma care in patients requiring surgery after multiple trauma. Emerg Med J 28: 300-304

Publisher's note Springer Nature remains neutral with regard to jurisdictional claims in published maps and institutional affiliations. 